\title{
Management of community-acquired pneumonia in the emergency department
}

\author{
Jerome A. Leis MD, Wayne L. Gold MD \\ See also practice article by Mumoli and Cei on page 560 and at www.cmaj.ca/lookup/doi/10.1503/cmaj.111256
}

\section{Community-acquired pneumonia is life-threatening and timely administra- tion of antibiotics is a goal of therapy}

In-hospital mortality associated with community-acquired pneumonia is comparable to that with acute myocardial infarction. ${ }^{1}$ Patients with suspected community-acquired pneumonia require urgent assessment and therapy; however, overemphasis on time to first dose of antibiotics may lead to unnecessary administration of antibiotics for noninfectious diseases. ${ }^{2}$ Because of a lack of convincing evidence, guidelines no longer specify a four-hour window for starting antibiotics for patients who require admission to hospital but instead recommend that the first dose be given in the emergency department. ${ }^{2,3}$

Severe disease requires early recognition and prompt referral to intensive care

Current guidelines recommend early referral to intensive care for patients with hypotension despite aggressive fluid resuscitation, hypothermia, elevated respiratory rate $(>30$ breaths $/ \mathrm{min}$ ), $\mathrm{PaO}_{2} / \mathrm{FiO}_{2}$ ratio $<250$, leukopenia $[<4 \times 10 \%$ L], multilobar consolidation, thrombocytopenia $\left(<100 \times 10^{9} / \mathrm{L}\right)$, confusion or uremia (blood urea nitrogen $\geq 20 \mathrm{mmol} / \mathrm{L}$ ). ${ }^{3}$ The presence of three or more of these criteria has recently been validated in a prospective observational trial for predicting need for intensive care. $^{4}$

\section{Pretreatment diagnostic tests should be requested on a case-by-case basis}

Routine cultures of blood and sputum are controversial because of their low yield and impact on treatment decisions. These tests are optional for outpatients; however, they should be ordered for patients who require admission to hospital, even in the absence of fever, if there is an increased risk of unusual or resistant pathogens that would require a change of empiric therapy or duration. This includes patients with severe community-acquired pneumonia, an immunocompromised state, alcohol abuse, structural lung disease, the presence of a pleural effusion and those who have recently travelled, as well as those for whom outpatient therapy has failed. ${ }^{3}$

Determining who can receive treatment as an outpatient should be guided by validated scoring systems

Predictive scores including the CURB65 (Confusion, Uremia, Respiratory rate, low Blood pressure, age $\geq 65$ years) or Pneumonia Severity Index are useful for identifying those at low risk of death who may be safely cared for as outpatients. ${ }^{3}$ A patient's oxygen saturation $\left(\mathrm{Sp}_{2}\right)$ on room air is increasingly recognized as a stand-alone criterion for discharge decisions. A recent prospective cohort study of 2923 patients with community-acquired pneumonia showed that those discharged from the emergency department with an $\mathrm{SpO}_{2}$ of less than $90 \%$ had increased combined 30day mortality and admission rates (odds ratio 1.7 ; $95 \%$ confidence interval $1.1-$ $2.8 ; p=0.032) .{ }^{5}$ When an $\mathrm{Sp}_{2}$ of $92 \%$ or greater was used, the association with adverse events was no longer present.

For references, please see Appendix 1, available at www.cmaj.ca/lookup/suppl /doi:10.1503/cmaj.111143/-/DC1

Competing interests: None declared.

This article has been peer reviewed.
Outpatient therapy should take into account risk factors for drugresistant Streptococcus pneumoniae

Patients with exposure to macrolides, trimethoprim-sulfamethoxazole or respiratory fluoroquinolones in the past three months are at least four times more likely than those without such exposure to be infected with a pneumococcal isolate that is resistant to the same class of antibiotics. ${ }^{6}$ Although macrolides are the recommended first-line agents for those treated as outpatients, patients with recent macrolide exposure or who live in regions with high rates of macrolide resistance $(>25 \%)$ should receive alternate forms of empiric therapy. ${ }^{3,6}$ Treatment options include the addition of amoxicillin or amoxicillin-clavulanate or monotherapy with a respiratory fluoroquinolone. ${ }^{3}$

Affiliation: From the Division of Infectious Diseases, University Health Network, Department of Medicine, University of Toronto, Toronto, Ont.

Correspondence to: Dr. Wayne L. Gold, wayne .gold@uhn.on.ca

CMAJ 2012. DOI:10.1503/cmaj.111143 